Revista Tecné, Episteme y Didaxis: TED. Año 2014, Número Extraordinario. ISSN Impreso: 0121-3814, ISSN web: 2323-0126

Memorias, Sexto Congreso Internacional sobre Formación de Profesores de Ciencias. 08 al 10 de octubre de 2014, Bogotá

\title{
Jerarquizar el Modelo Científico Escolar de Arribo sobre el origen de los terremotos: una herramienta para el Desarrollo Curricular
}

Faustinos Garrido, Maria de Lourdes'1, Rodríguez Pineda, Diana Patricia²

Categoría 2. Trabajo de investigación

\section{Resumen}

De acuerdo con López-Mota y Rodríguez-Pineda (2013) el Modelo Científico Escolar de Arribo (MCEA) orienta el diseño de las Estrategias Didácticas, mediante las cuales el profesor realiza el desarrollo curricular. En este trabajo se presenta el MCEA sobre el origen de los terremotos y la jerarquización del mismo, mediante la construcción de un Mapa de Diseño Curricular (MDC) permitiendo estructurar los elementos, relaciones y condiciones que lo constituyen; convirtiéndose este MDC (García-Martínez e Izquierdo, 2014) en la carta de navegación y herramienta clave para el diseño de una Estrategia Didáctica que permita abordar el tema de los terremotos en la escuela primaria. Lo anterior resulta relevante en la formación de profesores, si se concibe ésta, bajo la idea de que el profesor es el gestor en el aula mediante el desarrollo curricular.

\section{Palabras clave}

Modelo Científico Escolar de Arribo, Mapa de Diseño Curricular y Estrategia Didáctica

\section{Objetivo}

El objetivo de este trabajo, es mostrar la jerarquización del Modelo Científico Escolar de Arribo (MCEA) sobre el origen de los terremotos, que permite comprender las relaciones existentes entre los elementos, relaciones y condiciones de este modelo, considerando que la conexión entre ellos este clara para el diseño de una Estrategia Didáctica (ED). La estructuración y selección del MCEA nos permite ser coherentes con las actuales concepciones sobre la naturaleza de la ciencia (Sánchez Blanco y Valcárcel Pérez, 1993).

\footnotetext{
1 Universidad Pedagógica Nacional, Estudiante de Maestría, hlourdes_11@hotmail.com
}

2 Universidad Pedagógica Nacional, Profesora Titular, dpineda@upn.mx 
Revista Tecné, Episteme y Didaxis: TED. Año 2014, Número Extraordinario. ISSN Impreso: 0121-3814, ISSN web: 2323-0126

Memorias, Sexto Congreso Internacional sobre Formación de Profesores de Ciencias. 08 al 10 de octubre de 2014, Bogotá

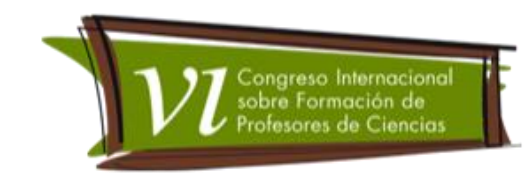

Por tanto, la construcción de un esquema conceptual (Sánchez Blanco y Valcárcel Pérez, 1993) y particularmente de un Mapa de Diseño Curricular (MDC) como lo han denominado García-Martínez e Izquierdo (2014) y García-Martínez, Mora y Enciso (2005), permite estructurar y delimitar el fenómeno de estudio así como decidir qué elementos tienen un carácter descriptivo, interpretativo y explicativo que nos permita conocer funciones características del modelo.

Es necesario reconocer que el uso de esquemas conceptuales -que son tan solo representaciones- permite reflejar los conceptos y relaciones más relevantes. Se asume que la construcción del MDC es una herramienta útil para el diseño de una ED.

\section{Marco Teórico}

De acuerdo con Rodríguez-Pineda, López-Mota, López y Flores (2013), el profesor puede llevar a cabo el desarrollo curricular, mediante el diseño de una ED y para ello "El Modelo Científico Escolar de Arribo es un dispositivo teórico-conceptualmetodológico en el ámbito de la investigación en didáctica de la ciencia, que permite orientar el diseño, la recolección de evidencias y su sistematización, así como la evaluación de una ED sustentada en la modelos y la modelización" (López-Mota y Rodríguez-Pineda, 2013: 2010). Como todo modelo científico es importante identificar en él los elementos, relaciones y condiciones o reglas de operación del mismo -de acuerdo con Lesh \& Doerr (2003) citados por Schwarz, et al. (2009).

Si bien lo anterior permite al docente tener una guía clara para el diseño de la ED y secuenciar las actividades -partiendo de lo simple a lo complejo y de lo concreto a lo abstracto (Sanmartí, 2002)-, también se requiere conocer claramente el Modelo Explicativo Inicial (MEI) de los alumnos, para lograr la transformación o evolución al modelo que se espera que arriben, de manera que incorporen elementos que no estaban presentes en sU MEI.

Para García-Martínez e Izquierdo (2014) el Mapa de Diseño Curricular es una herramienta de diseño curricular a nivel conceptual, que permite estructurar los elementos, relaciones y condiciones que constituyen el MCEA; convirtiéndose este MDC en la carta de navegación y herramienta clave para el diseño de una ED. Jerarquizar el MCEA permite orientar las actividades docentes y la evaluación en el aula. 
Revista Tecné, Episteme y Didaxis: TED. Año 2014, Número Extraordinario. ISSN Impreso: 0121-3814, ISSN web: 2323-0126

Memorias, Sexto Congreso Internacional sobre Formación de Profesores de Ciencias. 08 al 10 de octubre de 2014, Bogotá

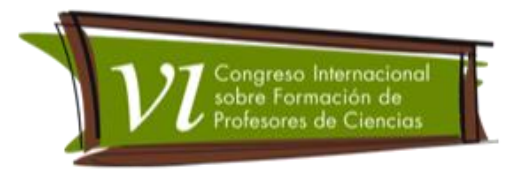

\section{Metodología}

El Modelo Científico Escolar de Arribo enlaza el Modelo Científico Erudito (MCE) y el Modelo Curricular (MCU) aportando elementos, relaciones y condiciones. El constructo del MCE procede de la revisión de libros especializados sobre la teoría de Tectónica de Placas (Cruz-Atienza, 2013; Tarbuk y Lutgens, 2008), siendo el modelo que explica al fenómeno del origen de los terremotos a partir de lo que dice la ciencia experta.

El Modelo Curricular se deriva de la revisión de los diferentes materiales curriculares vigentes de la Secretaria de Educación Pública (SEP) tales como: libro de texto de geografía quinto grado (SEP, 2013), Programa de Estudios de la Educación Básica Primaria (SEP, 2011), Atlas de México (SEP, 2013), Atlas Universal (SEP, 2013). A partir de estos materiales curriculares se pueden inferir los elementos, relaciones y condiciones del MCU.

Una vez construidos el MCE y el MCu y estando estos modelos organizados de manera homogénea (elementos, relaciones y condiciones) de tal manera que sean comparables (López-Mota y Rodríguez-Pineda, 2013), se procedió a construir el MCEA para explicar el fenómeno de los terremotos en la educación primaria.

Finalmente, siguiendo los planteamientos de Sánchez Blanco y Valcárcel Pérez (1993) y García-Martínez e Izquierdo (2014), se jerarquizan en un MDC los conceptos -elementos, relaciones y condiciones- del MCEA de la Tectónica de Placas.

\section{Resultados}

Una vez revisados los textos especializados sobre la Tectónica de Placas, se infirió el MCE, el cual se muestra en la tabla 1.

Tabla 1. Modelo Científico Erudito (MCE)

\begin{tabular}{|c|c|c|c|}
\hline Elementos & & Relaciones & Condiciones \\
\hline $\begin{array}{l}\text { Litosfera: } \\
\text { tectónicas } \\
\text { oceánico } \\
\text { Densidad } \\
\text { Espesor }\end{array}$ & $\begin{array}{r}\text { placas } \\
\text { y fondo }\end{array}$ & $\begin{array}{ll}\text { - } & \text { Isostasia } \\
\text { - } & \text { Interacción } \\
\text { - } & \text { Contacto por fricción } \\
\text { - } & \text { Flotación } \\
\text { - } & \text { Borde o límites de las } \\
& \text { placas } \\
\text { - } & \text { Fuerzas (esfuerzos) de }\end{array}$ & $\begin{array}{l}\text { - Deformación las rocas } \\
\text { - Flotación } \\
\text { - Flujo de calor } \\
\text { (conducción) } \\
\text { - Convección }\end{array}$ \\
\hline
\end{tabular}


Revista Tecné, Episteme y Didaxis: TED. Año 2014, Número Extraordinario. ISSN Impreso: 0121-3814, ISSN web: 2323-0126

Memorias, Sexto Congreso Internacional sobre Formación de Profesores de Ciencias. 08 al 10 de octubre de 2014, Bogotá
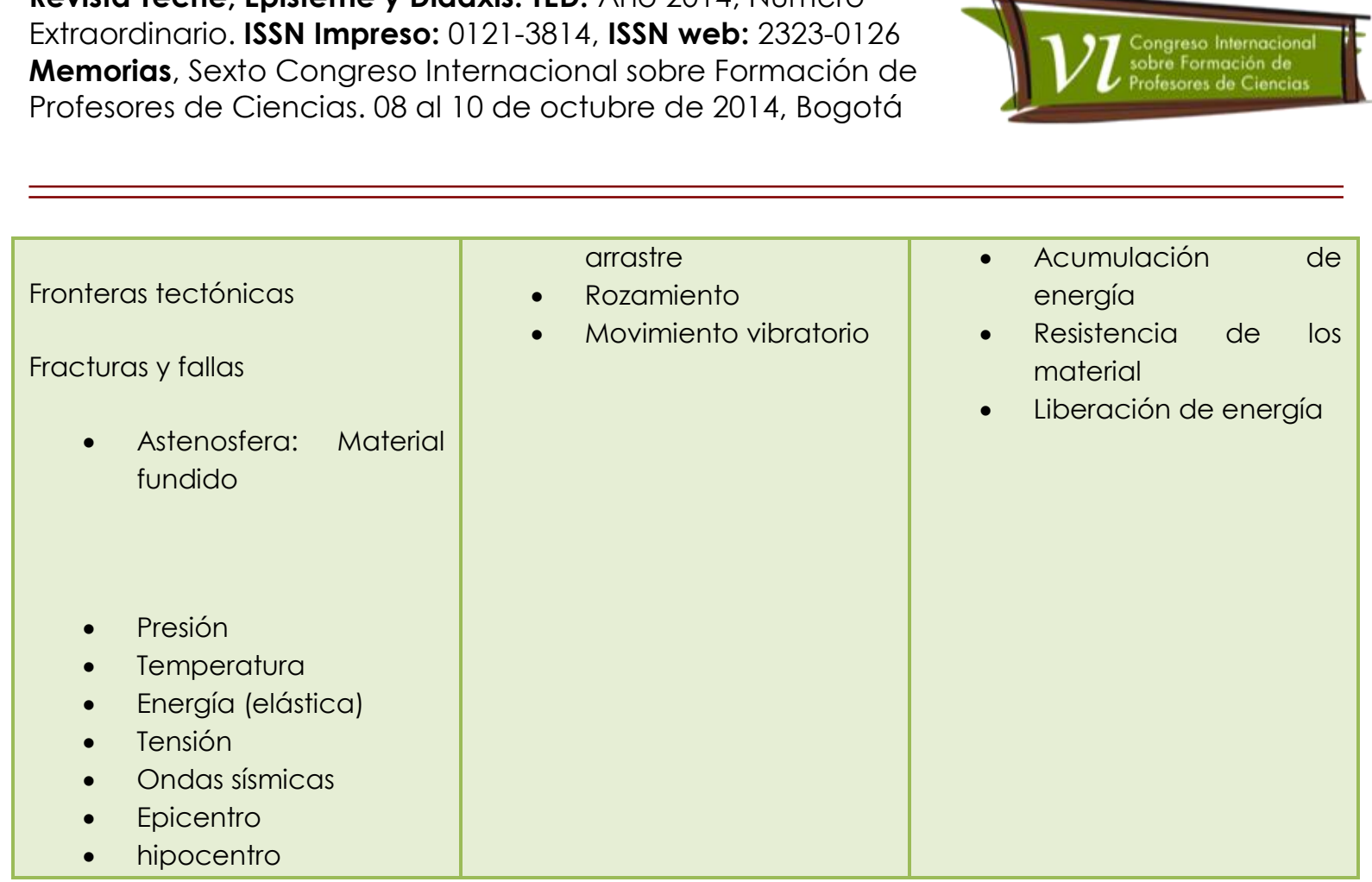

De igual manera, una vez revisados los materiales curriculares de la Educación Básica Primaria Educación Primaria, se infirió el MCu (ver tabla 2).

Tabla 2- Modelo Curricular (MCU)

- Capas internas de la tierra.

- Manto

- Corteza (Litosfera)

- Placas tectónicas

- Energía
- Flotación

- Desplazamiento

- Rozamiento

- Choque

- Movimiento
- Materiales fundidos

- Factores internos

- Liberación de energía

Una vez construidos el MCE y el MCU, se comparan y se construye el MCEA, teniendo presente que éste presenta los elementos, relaciones y condiciones, que serán la herramienta teórica que permita diseñar la ED para los alumnos de quinto grado de la educación primaria (ver tabla 3).

Tabla 3. Modelo Científico Escolar de Arribo (MCEA)

\begin{tabular}{|l|l|l|}
\hline \multicolumn{1}{|c|}{ Elementos } & Relaciones & Condiciones \\
\hline Litosfera: placas tectónicas & - Flotación & \\
& - Interacción \\
& - Fuerzas de arrastre & - Resistencia de los \\
\hline
\end{tabular}


Revista Tecné, Episteme y Didaxis: TED. Año 2014, Número Extraordinario. ISSN Impreso: 0121-3814, ISSN web: 2323-0126

Memorias, Sexto Congreso Internacional sobre Formación de Profesores de Ciencias. 08 al 10 de octubre de 2014, Bogotá

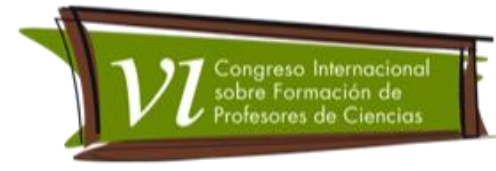

- Densidad

- Espesor

- Fronteras tectónicas

- Fractura o falla
- Contacto por fricción

- Rompimiento de la roca

- Movimientos sísmicos materiales

- Acumulación de energía elástica

- Liberación de energía

Astenosfera: Materiales

fundidos

La jerarquización del Modelo Científico Escolar de Arribo en el Mapa de Diseño Curricular permite organizar de manera clara, profunda y coherente, los elementos, relaciones y condiciones a un nivel conceptual que permiten orientar las actividades de la ED.

En el Mapa de Desarrollo Curricular sobre el origen de los terremotos para estudiantes de quinto grado de educación primaria que se presenta en la figura 1 , se presentan los elementos, relaciones y condiciones de menor a mayor jerarquía (de abajo hacia arriba), que se van organizan de acuerdo a la complejidad y abstracción para que sean enseñables y aprendibles.

En la parte superior del MDC -donde está la mayor complejidad y nivel de abstracción-, se encuentra la relación de interacción de las placas tectónicas, el contacto por fricción, fuerzas de arrastre, así como las condiciones de resistencia de los materiales, acumulación de energía provocando el rompimiento de la roca generando movimientos sísmicos.

La figura 1 presenta en la parte inferior del MDC los elementos, más concretos y simples como: Tierra (composición Física), Litosfera (Placas tectónicas: características como densidad, espesor, fracturas y fronteras tectónicas), Astenosfera (materiales semifundidos) y la relación de Flotación. 
Revista Tecné, Episteme y Didaxis: TED. Año 2014, Número

Extraordinario. ISSN Impreso: 0121-3814, ISSN web: 2323-0126

Memorias, Sexto Congreso Internacional sobre Formación de

Profesores de Ciencias. 08 al 10 de octubre de 2014, Bogotá

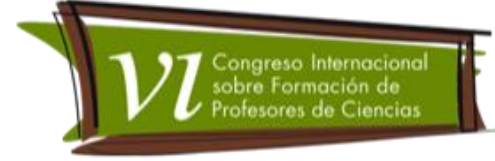

Figura 1. Mapa de Diseño Curricular sobre el origen de los terremotos para estudiantes de quinto grado de educación primaria

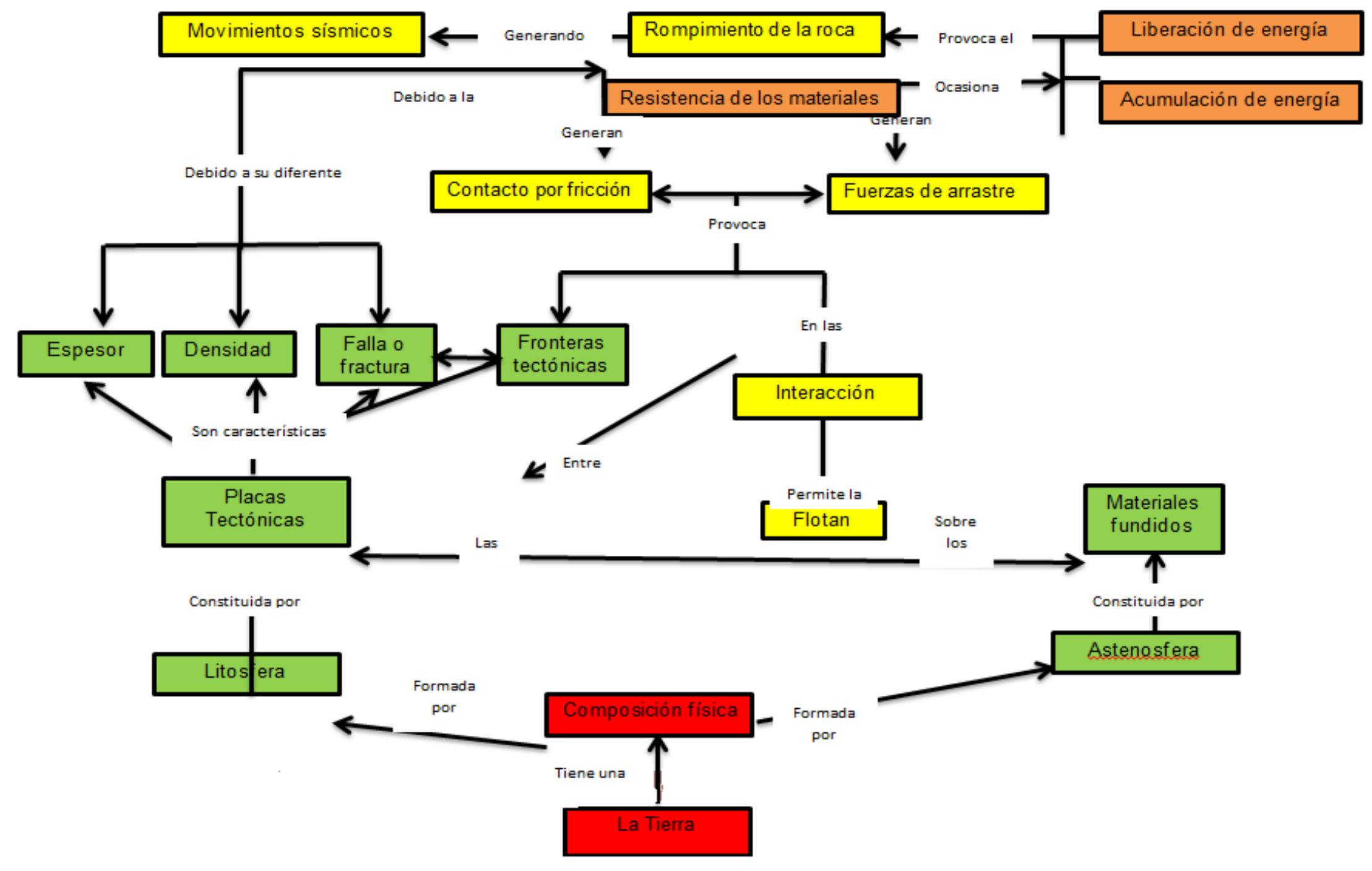


Revista Tecné, Episteme y Didaxis: TED. Año 2014, Número Extraordinario. ISSN Impreso: 0121-3814, ISSN web: 2323-0126

Memorias, Sexto Congreso Internacional sobre Formación de Profesores de Ciencias. 08 al 10 de octubre de 2014, Bogotá

\section{Conclusiones}

Este trabajo nos ha permitido estructura y delimitar el fenómeno del origen de los terremotos, permitiendo tener una mayor coherencia entre los elementos.

La jerarquización del MCEA en el MDC, nos brinda la posibilidad de organización de los elementos relaciones y condiciones de acuerdo al nivel de complejidad y abstracción para ser enseñables y aprendibles, permitiendo ver los niveles de complejidad y por tanto permite identificar las actividades apropiadas para la introducción de estos elementos, relaciones o condiciones del MCEA y secuenciar las actividades de la ED. Así como permitir los cortes necesarios, para ver la evolución de los modelos explicativos alcanzados durante la aplicación de la ED.

Ahora bien, si asumimos como lo plantean Rodríguez-Pineda et al. (2013), que la formación permanente de profesores debe estar sustentada en la idea de que el profesor es el gestor en el aula mediante el desarrollo curricular, resulta primordial la jerarquización del MCEA mediante el MDC para el diseño, aplicación y evaluación de ED, realizadas por ellos, es decir que este trabajo puede ser útil en la formación de profesores.

\section{Referencias Bibliográficas}

Cruz-Atienza, V.M. (2013). Los sismos: una amenaza cotidiana. Distrito Federal, México: La caja de cerrillos.

García-Martínez, A. e Izquierdo Aymerich, M. (2014). Contribución de la Historia de las Ciencias al desarrollo profesional de docentes universitarios. Enseñanza de las Ciencias, 32 (1), 265-281.

García-Martínez, A., Mora Penagos, W. y Enciso Galindo, S. (2005). La formación pedagógico didáctica del profesorado universitario de las áreas de ciencias naturales y tecnología. Enseñanza de las Ciencias (Número extra), 1 -5.

López-Mota, A. y Rodríguez-Pineda, D.P. (2013). Anclaje de los Modelos y la Modelización Científica en Estrategias Didácticas. Enseñanza de las Ciencias (Número extra), 2008 -2013.

Rodríguez-Pineda, D.P., López y Mota, A.D., López Becerra, C. y Flores López, M.L. (2013). El campo de Educación en Ciencias: una mirada desde la UPN. Revista Entre Maestr@s, 13 (46), 60-67. 
Revista Tecné, Episteme y Didaxis: TED. Año 2014, Número Extraordinario. ISSN Impreso: 0121-3814, ISSN web: 2323-0126

Memorias, Sexto Congreso Internacional sobre Formación de Profesores de Ciencias. 08 al 10 de octubre de 2014, Bogotá

Sánchez Blanco, G. y Valcárcel Pérez, M. (1993). Diseño de unidades didácticas en el área de ciencias experimentales. Enseñanza de las ciencias, 11 (1), 3344.

Sanmartí, N. (2002). Didáctica de las ciencias en la educación secundaria obligatoria. Madrid, España: Síntesis Educación.

Schwarz, C., Reiser, B., Davis, E., Kenyon, L., Achér, A., Fortus, D., Shwartz, Y., Hug, B. y Krajcik, J. (2009). Developing a learning progression for scientific modeling: making scientific modeling accessible and meaningful for learners. Journal of Research in Science Teaching, 46 (6,) 632-654.

Secretaría de Educación Pública (2011). Programas de Estudio 2011. Educación Básica Primaria. México: SEP.

Secretaría de Educación Pública (2011). Libro de texto de Geografía quinto grado 2013. Educación Básica Primaria. México: SEP.

Secretaría de Educación Pública (2011). Atlas de México quinto grado 2013. Educación Básica Primaria. México: SEP.

Secretaría de Educación Pública (2011). Atlas Universal de Sexto grado 2013. Educación Básica Primaria. México: SEP.

Tarbuk, E. y Lutgens, K. (2008). Ciencias de la Tierra: Una introducción a la geología física. España: Pearson. 